Bundesgesundheitsbl - Gesundheitsforsch Gesundheitsschutz 2006 · 49:485-486 DOI 10.1007/s00103-006-1277-9

๑) Springer Medizin Verlag 2006

\section{Vorschlag zur Gliederung von umweltmedizinischen Kasuistiken}

\author{
Mitteilung der Kommission „Methoden und \\ Qualitätssicherung in der Umweltmedizin"
}

Stellenwert von Kasuistiken in der Umweltmedizin

Gut dokumentierte klinische Fallbeschreibungen (empirisch-kasuistische Forschungsmethode) haben in der Medizin eine lange Tradition und wesentlich zur Entwicklung des medizinischen Fachwissens beigetragen. Sie lassen sich durch die moderne Epidemiologie oder Toxikologie nicht vollständig ersetzen. Während die Toxikologie ihr Augenmerk bevorzugt auf akut auftretende Wirkungen von Schadstoffen in hohen Konzentrationen richtet und in der Regel von dem Axiom ausgeht: „mehr Dosis = mehr Wirkung ", bevorzugen die Verfahren der Epidemiologie "häufige“ Reaktionen einer „durchschnittlichen“ Bevölkerung. Der unerwartete, seltene und ungewöhnliche „Fall“ fällt dabei häufig durch das Raster dieser beiden wissenschaftlich etablierten Methoden. Zwei Prozent einer Population, die bei Betrachtung z.B. des 98. Perzentils unberücksichtigt bleiben, sind, bezogen auf die Bevölkerung Deutschlands, immerhin fast 2 Millionen Individuen. Ebenso stellen Beobachtungen über die Wirkungen vieler Rausch- und Arzneimittel, bei denen niedrige Dosen qualitativ andere Wirkungen zeigen als hohe, die in der Toxikologie übliche Annahme einer monoton steigenden Dosis-Wirkungs.-Beziehung gelegentlich in Frage. Große interindividuelle Empfindlichkeitsunterschiede und intraindividuelle Variationen der DosisWirkungs-Beziehungen aufgrund von Gewöhnungs- und Sensibilisierungsphänome- nen spielen wohl auch bei der Wirkung von Umweltnoxen auf die Gesundheit des Menschen eine wichtige Rolle.

Die systematische Erfassung und Auswertung von Einzelbeobachtungen durch Ärzte in ihrer täglichen klinischen Praxis ist deshalb für die Umweltmedizin von wesentlicher Bedeutung. Es kommt darauf an, dass Ärzte in Klinik und Praxis ihre Aufmerksamkeit mehr als bisher auf die mögliche Verursachung der Beschwerden ihrer Patienten durch Umweltfaktoren richten. Das heißt: Sie sollten die von ihnen beobachteten Fälle im Hinblick auf Zusammenhänge zwischen objektivierbaren gesundheitlichen Störungen und der Exposition gegenüber Umweltnoxen vorurteilsfrei diagnostisch aufarbeiten, beschreiben und ihre Erfahrungen der wissenschaftlichen Öffentlichkeit zur Kenntnis bringen.

Eine systematische Dokumentation von Einzelbeobachtungen stoffbezogener Erkrankungen wurde bereits 1990 in die deutsche Chemikaliengesetzgebung eingeführt, was international ein Novum war. Die dahinter stehende Idee war, toxikologische Tierversuche durch Beobachtungen menschlicher Vergiftungsunfälle zu ergänzen.

\section{Grundsätze für eine rationale Fallbeschreibung in der Umweltmedizin}

Bei umweltmedizinischen Kasuistiken ist grundsätzlich nicht anders vorzugehen, als auch sonst bei Fallbeschreibungen in der klinischen Medizin. Wichtig ist, die
Gründe darzustellen, warum der Patient den Arzt aufsuchte. Hinzu kommen ein kurzer Abriss der diagnoseleitenden Fakten aus der Anamnese, pathologische Befunde der körperlichen Untersuchung, Indikation und Ergebnisse der durchgeführten Laboruntersuchungen und Funktionstests. Die möglichen Differentialdiagnosen sollten unter dem Bild der Symptome und Befunde diskutiert werden. Abschließend sollten die Beweggründe für die endgültige Diagnose dargestellt werden.

Umweltmedizinische Fallbeschreibungen weisen folgende zusätzliche Besonderheiten auf: Alle Hinweise, die vermuten lassen, dass Symptome und Gesundheitsbeeinträchtigungen auf eine Umweltverursachung zurückzuführen sind, werden vermerkt.

Die infrage kommende Noxe muss im Einzelnen geklärt werden. Dabei sind räumliche und zeitliche Bezüge der Symptome im Hinblick auf Belastung mit der angeschuldigten Noxe von Bedeutung. Ein wichtiger Hinweis auf einen Zusammenhang zwischen Exposition und einer gesundheitlichen Wirkung ist, wenn sich nachweisen lässt, dass eine Verminderung der Exposition oder eine erneute Belastung zu einer Änderung der Symptomatologie führt.

Für die umweltmedizinische Kasuistik sind besonders auch Ergebnisse eines Ambient- und/oder Human-Biomonitorings von Bedeutung. Auf der Basis repräsentativer Messungen der äußeren Exposition (Raumluft, Konzentrationen der Noxe in 
Nahrung, Kleidung etc.) bzw. der inneren Exposition (Konzentration der Noxe in den Körpermedien) ist im Prinzip eine quantitative Expositionsabschätzung möglich. Jedoch müssen Monitoringdaten im Hinblick auf ihre Relevanz für eine Kausalitätsbeurteilung kritisch hinterfragt werden. Die hier zu stellenden Fragen zielen auf die Möglichkeit und das Ausmaß der Exposition. Wurde der richtige Parameter bestimmt? Wurde richtig und mit adäquater Messgenauigkeit analysiert? Und schließlich wurde richtig interpretiert (siehe auch $[1,2])$ ?

In der Fallbeschreibung sollte diskutiert werden, ob nach der wissenschaftlichen toxikologischen Erkenntnislage überhaupt ein Kausalzusammenhang zwischen Noxe und Symptomatik denkbar ist (z.B. passt die Symptomatik zur Noxe?). Die Kasuistik sollte mit einer abschließenden Einschätzung enden, aus der hervorgeht, mit welcher Wahrscheinlichkeit ein umweltmedizinischer Kausalzusammenhang vorliegt. Dies kann nach dem Bewertungsschema erfolgen: Kausalzusammenhang möglich - wahrscheinlich - sicher oder nicht bewertbar.

In jedem Falle sind aber gut dokumentierte Beschreibungen von außerordentlichen und überraschenden Fällen und Befunden besonders wertvoll. Die Kommission möchte deshalb alle umweltmedizinisch arbeitenden Kollegen ermuntern, solche Fallbeschreibungen $\mathrm{zu}$ veröffentlichen.

\section{Gliederungsvorschlag \\ für eine Kasuistik}

Ein lege artis dokumentierter Fall in der Umweltmedizin sollte enthalten:

- Angaben zu(r) Person(en), wie Alter und Geschlecht, Berufsanamnese, psychosoziale Situation, Lebensgewohnheiten (Rauchen, Alkohol),

- Grund für Arztkontakt (Anliegen),

- gegebenenfalls Vorerkrankungen,

- bisher beobachtete Unverträglichkeiten (Idiosynkrasien, Allergien),

- Angaben zu den Umständen der Exposition (z.B. Ort, beruflich/privat/Umwelt),

- Angaben zur Noxe (z.B. Chemikalie) oder Inhaltsangaben des Produktes, mit dem der Patient in Kontakt kam,

- Angaben zur aufgenommenen Menge,

- Angaben zum Aufnahmeweg (oral, inhalativ, dermal oder kombiniert),
- bei akuter Exposition: kurze Darstellung des Ereignisses/Unfall mit Problemstellung und Fallausgang,

- bei längerer Exposition: Zeitpunkt der Exposition, Beginn und Dauer,

- Ergebnisse von Ambient-MonitoringUntersuchungen,

- Ergebnisse von Human-Biomonitoring-Untersuchungen,

- Angaben zur Symptomatik,

- Beschreibung der Symptome (Anamnese und klinische Befunde),

- Angaben zum zeitlichen Verlauf der Symptomatik,

- räumliche und zeitliche Beziehung der Symptomatik zur Exposition,

- Ergebnisse von Funktionsuntersuchungen (z.B. darstellende Verfahren, Lungenfunktion, Belastungsuntersuchungen),

- Ergebnisse von biochemischen Laboruntersuchungen,

- Angaben zur durchgeführten Therapie und deren Konsequenzen,

- Angaben zum Fallausgang,

- Differenzialdiagnosen,

- toxikologische Überlegungen, Literaturbezug,

- zusammenfassende, kritische Bewertung des Falles und Einordnung in das oben vorgeschlagene Bewertungsschema: Kausalzusammenhang möglich - wahrscheinlich - sicher - nicht bewertbar.

\section{Federführung}

Prof. Dr. Dr. med. A. Kappos (Kommissionsmitglied), Frankfurt am Main, Dr. med. A. Beyer (Kommissionsmitglied), Umweltmedizinische Ambulanz Berlin-Steglitz/ Zehlendorf, Dr. med. A. Hahn (ständiger Gast der Kommission), Bundesinstitut für Risikobewertung, Berlin

\section{RKI-Kommission „Methoden und Qualitätssicherung in der Umweltmedizin"}

\section{Weitere Kommissionsmitglieder}

Prof. Dr. rer. nat. W. Dott (Universitätsklinikum Aachen, Institut für Hygiene und Umweltmedizin), Prof. Dr. med. H. Drexler (Friedrich-Alexander-Universität Erlangen-Nürnberg, Institut für Arbeits-, Sozial- und Umweltmedizin), Prof. Dr. med. H. Dunkelberg (Universität Göttingen, Abt. Allg. Hygiene und Umweltmedizin), Prof. Dr. med. Th. Eikmann (Universität Gießen, Institut für Hygiene und Umweltmedizin), Dr. med. B. Heinzow (Landesamt für Gesundheit und Arbeitssicherheit des Landes Schleswig-Holstein, Dezernat Umweltbezogener Gesundheitsschutz, Kiel), Prof. Dr. C. Hornberg (Universität Bielefeld, Fakultät für Gesundheitswissenschaften), Institut für Innenraum- und Umwelttoxikologie), Prof. Dr. med. K. E. von Mühlendahl (Kinderhospital Osnabrück, Gemeinnützige Kinderumwelt GmbH), Prof. Dr. med. D. Nowak (LMU München, Klinikum Innenstadt, Institut u. Poliklinik für Arbeits- und Umweltmedizin), PD Dr. med. F.-A. Pitten (Institut für Krankenhaushygiene und Infektionskontrolle GbR, Gießen), Dr. med. W. Stück (Ökologischer Ärztebund/ISDE, Koblenz), Prof. Dr. M. Schwenk (Tübingen), Dr. med. R. Suchenwirth (Niedersächsisches Landesgesundheitsamt, Abt. Umweltmedizin/Epidemiologie, Hannover), Prof. Dr. med. M. Wilhelm (Ruhr-Universität Bochum, Hygiene, Sozial- und Umweltmedizin).

\section{Ständige Gäste}

\section{S. Strecker}

(Bundesministerium für Gesundheit,Bonn), Dr. med. N. Englert

(Umweltbundesamt, Berlin).

\section{Geschäftsstelle im RKI}

Dr. med. D. Eis (Geschäftsführer), Dr. med. U. Wolf.

\section{Literatur}

1. HBM-Kommission (1996) Konzept der Referenzund Human-Biomonitoring-Werte (HBM) in der Umweltmedizin. Bundesgesundhbl Gesundheitsforsch Gesundheitsschutz 39: 221-224

2. HBM-Kommission (1996) Qualitätssicherung beim Human-Biomonitoring. Bundesgesundhbl Gesundheitsforsch Gesundheitsschutz 39: 216-221 